\title{
Pairing of few Fermi atoms in one dimension
}

\author{
Pino D'Amico* and Massimo Rontan † $^{*}$ \\ CNR-NANO Research Center S3, Via Campi 213/a, 41125 Modena, Italy
}

\begin{abstract}
We study a few Fermi atoms interacting through attractive contact forces in a one-dimensional trap by means of numerical exact diagonalization. From the combined analysis of energies and wave functions of correlated ground and excited states we find evidence of BCS-like pairing even for very few atoms. For moderate interaction strength, we reproduce the even-odd oscillation of the separation energy observed in [G. Zürn, A. N. Wenz, S. Murmann, A. Bergschneider, T. Lompe, and S. Jochim, Phys. Rev. Lett. 111, 175302 (2013)]. For strong interatomic attraction the arrangement of dimers in the trap differs from the homogeneous case as a consequence of Pauli blockade in real space.
\end{abstract}

PACS numbers: 67.85.Lm, 31.15.ac, 03.75.Ss, 74.20.Fg

\section{INTRODUCTION}

Pairing between fermions is a basic phenomenon emerging in quantum degenerate systems as diverse as electrons in metals [1], protons and neutrons in nuclei [2, 3] and neutron stars [4, [5, ${ }^{3} \mathrm{He}$ atoms [6], electrons and holes in semiconductors 7], cold atoms confined in magneto-optical traps [6, 8-12]. In nuclei, pairing enhances the stability of isotopes with an even number of constituents, reaching the maximum at the closure of an energy shell [2, 3, 13 -15]. In metals, electrons of opposite spins form Cooper pairs that condense in the superconducting phase, as explained by the weak-coupling theory by Bardeen, Cooper and Schrieffer (BCS) [16].

Experiments with cold Fermi atoms provide unprecedented control on both the shape of the trap confinement potential and the interatomic interaction strength - the latter by sweeping a magnetic offset field through a Feshbach resonance [17]. This enables novel possibilities, like to explore the transition from BCS-like superfluidity to Bose-Einstein condensation (BEC) of strongly bound atom dimers $8-10,18,19]$, to control the atom number $N$ with unit precision - down to the empty-trap limit 20 23], as well as to change the dimensionality of the system [11, 24 29].

In these tunable traps, the pairing gap $\Delta$ - the order parameter of the superfluid phase - may deviate from the expectations for homogeneous systems and exhibit a significant dependence on the atom number $N$ as well as on the dimensionality $d$ [30 32]. This is seen from BCS gap equation, which allows for a finite value of $\Delta$ provided the density of states $g(\varepsilon)$ is large at the Fermi surface $\varepsilon_{F}$ ( $\varepsilon$ is the energy reckoned from the bottom of the trap). This density is enhanced by the occurrence of energy shell degeneracies at higher dimensions, depending on $d$ as $g(\varepsilon) \sim \varepsilon^{d-1}$ on a coarse-grain energy scale [33]. Therefore, pairing is harder to accomplish at lower dimensions as it requires stronger inter-species attraction.

\footnotetext{
* pino.damico@nano.cnr.it

$\dagger$ massimo.rontani@nano.cnr.it
}

Besides, the spiked features of $g(\varepsilon)$ on the fine energy scale might make $\Delta$ strongly fluctuate when filling successive shells.

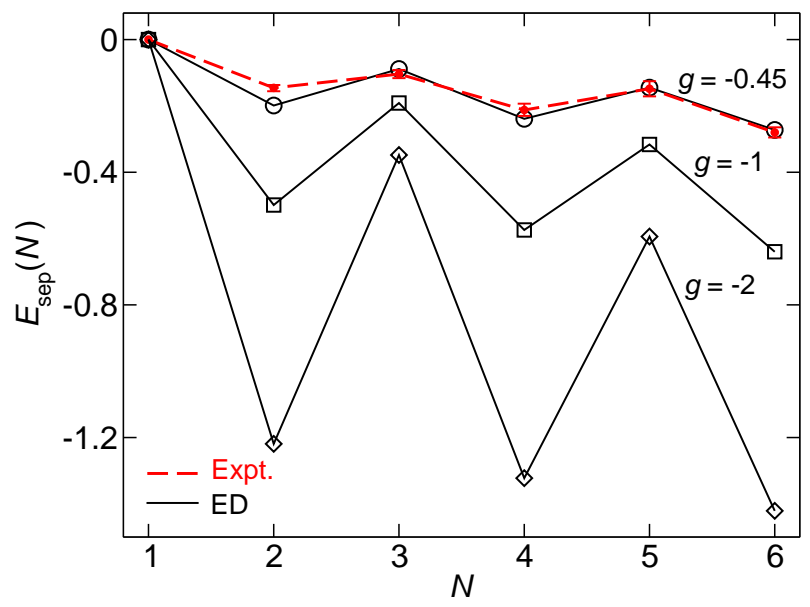

Figure 1. (color online) Separation energy $E_{\mathrm{sep}}(N)$ vs atom number $N$. Black circles, squares, diamonds with solid lines correspond to $g=-0.45,-1,-2$, respectively. Red [gray] circles with error bars and dashed lines are the measured data reported in [22]. The energy unit is $\hbar \omega$. Lines are guides to the eye.

A recent experiment by the Heidelberg group seems at odds with these expectations 22]. The magneto-optical trap was effectively one-dimensional (1D) as the aspect ratio was $1: 10$, the Fermi energy $\varepsilon_{F}$ was comparable to the longitudinal oscillator spacing, and the temperature was around half the Fermi temperature [20]. The trapping potential was deformed to measure the time spent by ${ }^{6} \mathrm{Li}$ atoms to tunnel out of the trap. This decay time was then linked to the separation energy of the system with $N$ fermions [34, 35], which exhibited a regular evenodd oscillation vs $N$ for moderate attraction strength and very small atom number, $N \leq 6$, as shown in Fig. 1)(dots with error bars and dashed lines). This alternate staggering was attributed to pairing, in analogy with similar data for neutron separation experiments in nuclei [3] . Intriguingly, BCS theory predicts that the pairing gap 
$\Delta$ vanishes exponentially with the interaction (see violet [gray] curve in Fig. 3).

These findings call for a theoretical analysis beyond mean-field BCS level, to take into account both finite-size fluctuations [36 41] and correlations at all orders. The problem of 1D Fermi gas with short-range interactions was solved exactly only for the homogeneous system, through either Bethe ansatz 42 45] (known as GaudinYang model for attractive interactions) or mapping to the Luttinger Hamiltonian [46 48]. Therefore, available results [45, 49 53] are useless for the harmonic trap analyzed here. On the other hand, the small number of ${ }^{6} \mathrm{Li}$ atoms studied in 22] allows for comparison with numerical exact diagonalization (ED), which provides energies and wave functions of both ground and excited states [54 61], whereas quantum Monte Carlo simulations are restricted to the ground state [12, 62 66].

In this paper we investigate theoretically the pairing between a few Fermi atoms populating a 1D harmonic trap. From the analysis of both ground- and excited-state ED energies we find that the pairing gap $\Delta$ is well defined even at small $N$, recovering the measured even-odd effect (black circles and solid lines in Fig. 1). The ED wave function is significantly affected by interaction already at moderate coupling strength, close to the regime achieved in the experiment 22]. For strongly bound dimers, the pair wave function exhibits a peculiar $N$-dependent spatial modulation that is absent in the bulk. This unexpected behavior - a manifestation of Pauli blockade in real space - may be observed using time-of-flight techniques.

The structure of this paper is as follows. We introduce the system Hamiltonian and the ED method in Sec. III Then we compare the ED separation energy with the measured data, also in connection with the fundamental energy gap (Sec. III). We evaluate the pairing gap $\Delta$ in two complementary ways, considering both ground-state energies by changing $N$ and excited-state energies for fixed $N$ (Sec. IV). We access the correlated ground state by computing the pair correlation function $G(x)$, which allows us to estimate the size of Cooper pairs (Sec. V). We eventually focus on the BEC-like regime of strong attraction, showing that $G(x)$ departs from the bulk behaviour due to Pauli blockade in real space (Sec. VI). After Conclusions, Appendix \$ illustrates the derivation of the bulk pair correlation function $G(x)$ plotted in Fig. 8(f).

\section{EXACT DIAGONALIZATION}

We consider $N$ atoms of spin $1 / 2$ confined in a $1 \mathrm{D}$ harmonic trap of frequency $\omega$ and interacting through an attractive contact force,

$$
H=\sum_{i=1}^{N}\left[\frac{p_{i}^{2}}{2 m}+\frac{1}{2} m \omega^{2} x_{i}^{2}\right]+g^{\prime} \sum_{i<j} \delta\left(x_{i}-x_{j}\right),
$$

where $g^{\prime}<0$ is the coupling constant and $m$ is the atom mass. Throughout this article we use $\hbar \omega$ as energy unit and $\ell=(\hbar / m \omega)^{1 / 2}$ as length unit, hence the dimensionless coupling constant is $g=g^{\prime} /(\hbar \omega \ell)$.

The ED wave function is the superposition of those Slater determinants obtained by filling the lowest $N_{\text {orb }}$ harmonic-oscillator orbitals with $N$ fermions in all possible ways (also known as full configuration interaction [54]). In this Fock space the Hamiltonian (10) is a sparse matrix, with blocks labeled by the total spin projection $S_{z}$, parity, and $N$. The maximum linear size of the eigenvalue problem (for $N=6$ and $N_{\text {orb }}=25$ ) is 2,644,928, which we solve with the home-built parallel code DONRODRIGO 54]. The ED convergence is demanding in the present attractive regime, as the method just mimics the cusp of the exact wave function induced by the contact interaction [61]. Therefore, the choice of $N_{\text {orb }}$ is the trade-off between accuracy and computational load, the Fock space size scaling exponentially with $N$. Here we used $N_{\text {orb }}=25$, with an error on the ground state absolute interaction energy of 5.6, 17, $22 \%$ for $g=-1$, $-2,-3$, respectively, and $N_{\text {orb }}=50$ in the illustrative case $N=3$ and $g=-4$ of Fig. 8 , which gives an error of $18 \%$. The accuracy on $\Delta$ is much higher due to mutual cancellation of systematic errors.

\section{SEPARATION ENERGY}

The key quantity we obtain from ED ground-state energies $E_{0}(N)$ is the chemical potential

$$
\Delta_{1}(N)=E_{0}(N)-E_{0}(N-1) .
$$

This is the lowest resonating energy of the $N$ th atom tunneling out of the trap while leaving the other $N-1$ atoms in the trap in their ground state [34, 35, 56]. The separation energy $E_{\mathrm{sep}}(N)$ is the net interaction energy contributing to $\Delta_{1}$,

$$
E_{\text {sep }}(N)=\Delta_{1}(N)-\Delta_{1}^{*}(N),
$$

with $\Delta_{1}^{*}$ being the chemical potential in the absence of interaction. The magnitude of $E_{\mathrm{sep}}$ is the contribution to the ionization energy due to interatomic attraction.

Figure 1 shows even-odd oscillations of $E_{\text {sep }}$ as the trap is filled with atoms. The ED spectrum (black circles with solid lines) fits well the measured data of [22] (red [gray] circles with error bars and dashed lines) for $g=-0.45$. This value reasonably compares with the experimental estimate of $g \sim-0.9$ (in our units), as the trap was strongly deformed with respect to the harmonic potential to allow the escape of atoms [67]. A possible reason for the residual mismatch between theory and experiment is the anharmonicity of the actual energy spacing in the trap.

In Fig. 1 both peak-to-valley ratios and magnitudes of absolute minima increase with attraction strength. Besides, the minima are deeper at higher atom numbers. 
These features are consistent with a BCS-like scenario, since: (i) the BCS ground state is more stable for even $N$, as all atoms are paired (ii) its energy gain increases with $N$-a signature of collective effect.

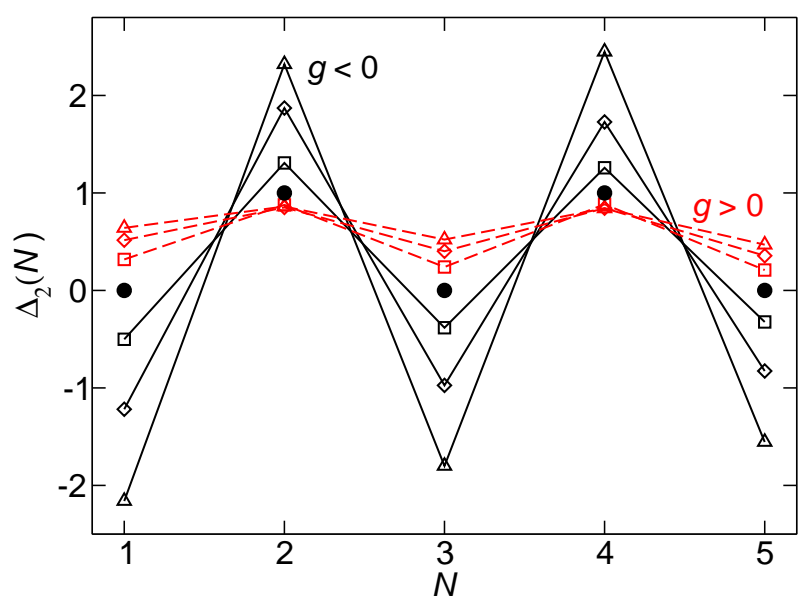

Figure 2. (color online) Fundamental energy gap $\Delta_{2}(N)$ vs atom number $N$. The black (red [gray]) color with solid (dashed) lines points to attractive (repulsive) interaction. Squares, diamonds, triangles correspond to $|g|=1,2,3$ respectively. The filled black circles are noninteracting data $(g=0)$. The energy unit is $\hbar \omega$. Lines are guides to the eye.

However, the observed even-odd oscillation of $E_{\text {sep }}$ might have a different explanation, being simply due to the filling of successive twofold degenerate trap orbitals. To clarify this matter we plot in Fig. 2 the fundamental energy gap

$$
\Delta_{2}(N)=\Delta_{1}(N+1)-\Delta_{1}(N)
$$

which is the difference between the tunneling energies of the atom added to and removed from the trap [56]. Here, it is instructive to consider repulsive (red [gray] symbols with dashed lines) interactions as well as attractive forces (black symbols with solid lines), since in both cases $\Delta_{2}$ exhibits an even-odd oscillation. At small coupling $(|g|=1$, square symbols) both patterns slightly deviate from the staggering noninteracting sequence (filled black circles), hence $\Delta_{2} \approx 1$ for even $N$ and $\approx 0$ for odd $N$, the energy separation between consecutive orbital levels being unity. As $|g|$ increases $\Delta_{2}$ changes qualitatively depending on the interaction sign. Strong repulsive interactions wash out the staggering of $\Delta_{2}$, which tends to a homogeneous positive value [58]. On the contrary, strong attractive forces enhance even-odd oscillations, suggesting BCS-like pairing. Indeed, if $N$ is even, all atoms form singlet pairs and a large amount of positive energy $\Delta_{2}$ is required to add one unpaired atom. For odd $N$, the fundamental gap $\Delta_{2}(N)$ is large and negative, since energy is gained by pairing with an opposite-spin atom.

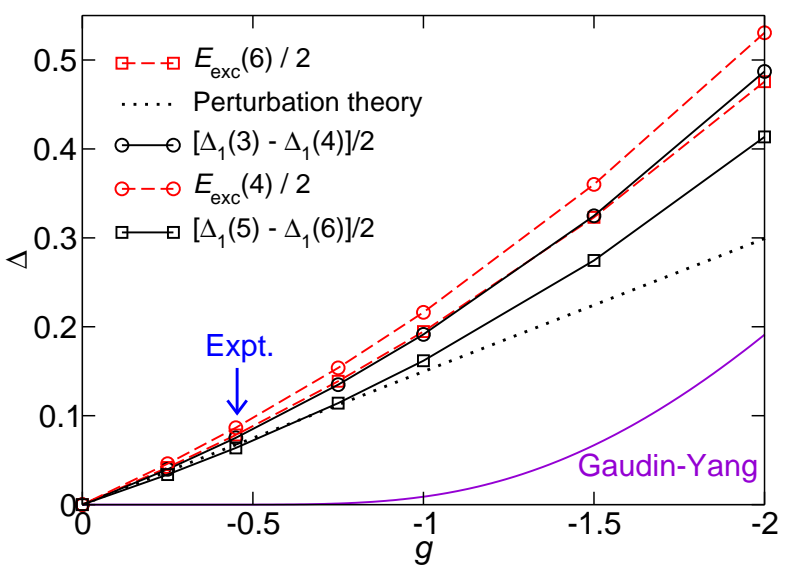

Figure 3. (color online) Complementary estimates of the pairing gap $\Delta$ vs interaction strength $g$. The violet [gray] curve is the exact prediction of Gaudin-Yang model in the BCS limit with $\varepsilon_{F}=3 / 2=\Delta_{1}^{*}(N=4)$. The dotted line is the prediction by perturbation theory whereas the remaining solid and dashed lines are guides to the eye. We use $\hbar \omega$ as energy unit and $\ell=(\hbar / m \omega)^{1 / 2}$ as length unit, hence the dimensionless coupling constant is $g=g^{\prime} /(\hbar \omega \ell)$.

\section{PAIRING GAP}

The computation of $\Delta_{1}$ allows us to evaluate the pairing gap $\Delta$ from two consecutive chemical potentials,

$$
\Delta=\frac{\Delta_{1}(N)-\Delta_{1}(N+1)}{2},
$$

with $N$ odd [12, 56]. Here the sign change is due to the staggering of $\Delta_{1}$, which alternately points to an energy expense and gain respectively for adding an unpaired atom and matching all pairs. Reassuringly, the pairing gaps $\Delta$ obtained for $N=3$ (black circles and solid lines in Fig. 3) and $N=5$ (black squares and solid lines) exhibit a similar dependence on $g$, coinciding within $15 \%$ at worse at large interaction strength $g=-2$. We see that $\Delta$ is a convex function of $g$, smoothly rising up to the value $\Delta \sim 0.5$, which is of the order of level spacing. The behavior of $\Delta$ is similar to that predicted in 2D [56] and 3D [65] for few atoms, suggesting that intra- and intershell contributions to pairing [30 32 ] are comparable.

A complementary study of the paring gap $\Delta$ relies on the ED excitation spectrum for fixed $N$. For the sake of clarity, we first focus on the paradigmatic case $N=2$, whose exact solution is known analytically [61, 68]. The low-lying energy spectrum for relative motion is shown in Fig. 4(b), limitedly to negative interaction strength $g$. There are two distinct families of energies branches, differing in orbital parity. The lines that vary with $g$ correspond to states that are even under particle exchange and hence associated with atoms of opposite spin, with $S_{z}=0$. The horizontal lines, independent from $g$, are the energies of two atoms of like spins with $S_{z}= \pm 1$, whose contact interaction is void as the orbital wave function is odd. Note that, in this odd sector, energy levels are 

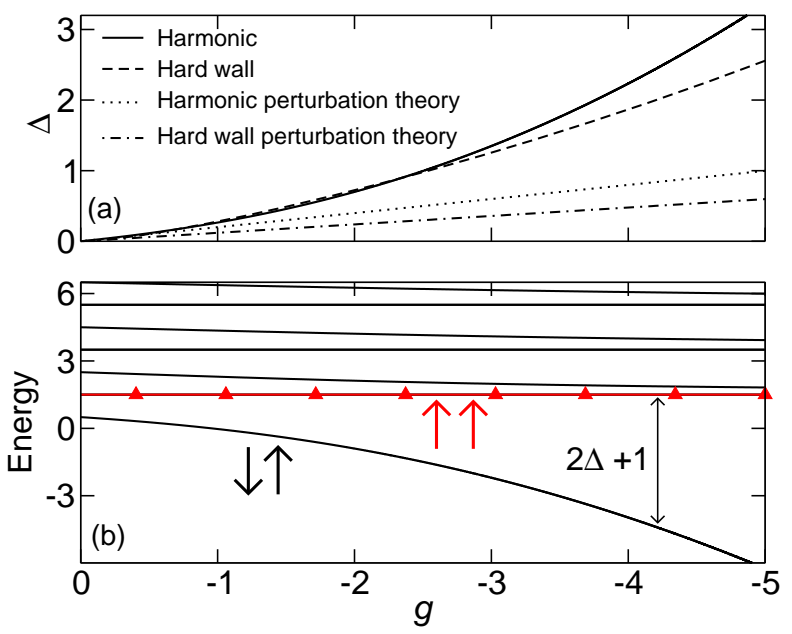

Figure 4. (color online) Extraction of the pairing gap $\Delta$ from the excitation spectrum of two atoms. (a) Paring gap $\Delta$ vs interaction strength $g$. The solid (dashed) curve refers to the exact result for two fermions with parallel spins in a harmonic (hard-wall) trap, with the harmonic oscillator length $\ell=(\hbar / m \omega)^{1 / 2}$ being equal to the width of the hard-wall quantum well. Dotted and dashed-dotted lines are the predictions of perturbation theory at first order in $g$ for the harmonic and hard-wall traps, respectively. (b) Low-lying energy spectrum of two-fermions in the harmonic trap in the relative frame vs g. $\Delta$ is inferred from the spin excitation gap separating the two lowest energy branches, which are respectively the lowest black line (balanced system with $S_{z}=0$ ) and the red (gray) line with triangles (unbalanced system with $S_{z}= \pm 1$ ). The energy unit is $\hbar \omega$ and the dimensionless coupling constant is $g=g^{\prime} /(\hbar \omega \ell)$.

degenerate with multiple center-of-mass excitations.

We link the paring gap $\Delta$ to the spin excitation gap 12, 51], which here is the energy difference between the two lowest energy branches, highlighted in Fig. 4(b). Clearly, we require $\Delta$ to vanish in the noninteracting limit $g \rightarrow 0$. Therefore, we subtract from the excitation gap a residual excitation energy quantum, which is unrelated to interactions and absent in the bulk. The remaining excitation gap is expected to be twice the gap $\Delta$ for BCS-like pairing, since the spin flip leaves two atoms unpaired [12, 15, 56].

The extracted value of $\Delta$ for $N=2$, shown in Fig. 4 (a) as a solid line, compares well with similar data obtained for higher atom numbers, as illustrated in Figs. 3 and 5 . In Fig. 5 we plot the lowest excitation energies $E_{\text {exc }}(N)$ of the system with $N=4$ (black lines) and $N=6$ (red [gray] dashed lines) and $S_{z}=0$, i.e., balanced spin population. Here we have referenced all energies to the ground state after subtracting the first center-of-mass excitation quantum. Again, multiple center-of-mass excitations are unrelated to atom-atom correlations and hence independent from $g$, as shown in Fig. 5 for the second excitation quantum. As the interaction strength $|g|$ increases an energy gap develops generically, since pairs must be broken

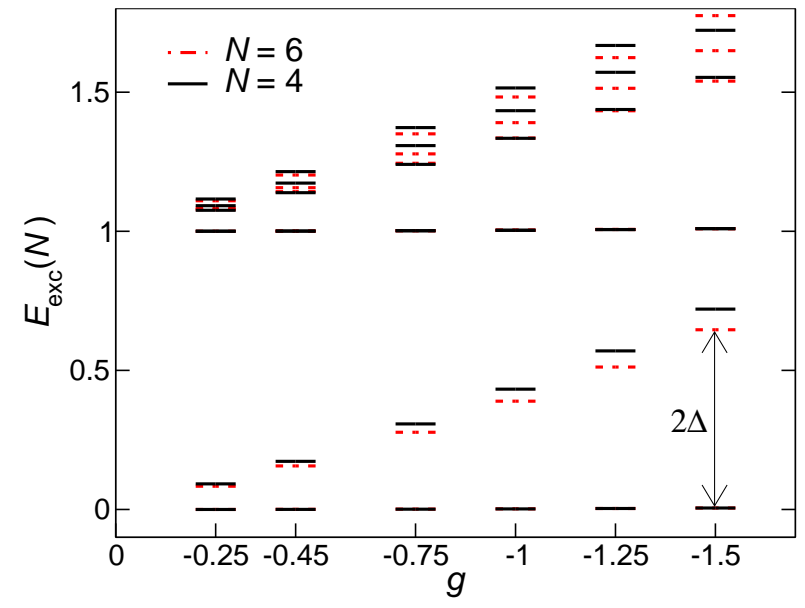

Figure 5. (color online) Excitation energies $E_{\text {exc }}(N)$ for $N=4$ (black lines) and $N=6$ (red [gray] dashed lines) vs $g$ for balanced spin population $\left(S_{z}=0\right)$. Energies were referenced to the ground state after subtracting the first center-of-mass excitation quantum. The two lowest branches are degenerate with those of the unbalanced system with $S_{z}= \pm 1$. We use $\hbar \omega$ as energy unit and $\ell=(\hbar / m \omega)^{1 / 2}$ as length unit, hence the dimensionless coupling constant is $g=g^{\prime} /(\hbar \omega \ell)$.

to excite the system. We find that, due to the symmetry of Hamiltonian (1), the two lowest excitations shown in Fig. [5]are degenerate with those obtained by flipping one atom spin $\left(S_{z}= \pm 1\right)$, as they are connected by a rotation in spin space. Therefore, we take these excitations to be twice the gap $\Delta$ (label in Fig. 5).

The estimate of $\Delta$ extracted from the excitation spectrum of Fig. 15 is plotted in Fig. 3 for $N=6$ (red [gray] squares and dashed lines) and $N=4$ (red [gray] circles and dashed lines). The good overall matching between these excitation gaps and the staggering-energy gaps discussed before (black symbols and solid lines) shows that a BCS-like pairing gap $\Delta$ emerges already for very few fermions, being relatively insensitive to finite-size fluctuations.

However, the magnitude of $\Delta$ significantly exceeds the BCS bulk value

$$
\Delta=\frac{8}{\pi} \varepsilon_{F} \sqrt{\frac{|\gamma|}{\pi}} \exp \left(-\frac{\pi^{2}}{2|\gamma|}\right)
$$

(in standard units), which is shown by the violet (gray) curve in Fig. 3. being the exact solution of GaudinYang model in the limit $\gamma \rightarrow 0-[42$, 51]. This discrepacy hardly depends on the Fermi energy $\varepsilon_{F}$ that enters the coupling constant $\gamma=(g \pi / \hbar)\left(m / 8 \varepsilon_{F}\right)^{1 / 2}$ [here $\left.\varepsilon_{F}=3 / 2=\Delta_{1}^{*}(N=4)\right]$, since in the bulk $\Delta$ vanishes exponentially - a non-perturbative result - whereas in the trap $\Delta$ scales almost linearly with $g$ up to $g \approx-1$.

This latter trend is well reproduced by perturbation theory, as shown by the dotted line in Fig. 3, using the estimate $\Delta=-3 g /(8 \sqrt{2 \pi})$, which is obtained by first averaging the interaction over the noninteracting ground 
states and then using these energy corrections for the staggering-energy definition (5) of $\Delta$ with $N=3$. Therefore, for the experiment 22] (arrow in Fig. 3), the wave function is substantially unaffected by interatomic correlations. For stronger interactions $\Delta$ significantly deviates from linearity, as a consequence of co-operative effects.

We attribute the departure of the functional form of $\Delta$ from the bulk exponential behaviour (6) to a genuine manifestation of few-body physics. In fact, whereas perturbation theory converges for the finite system, at least for small values of $g$, the Gaudin-Yang expression (6) is not analytic for $g \rightarrow 0^{-}$as a consequence of the divergence of perturbation theory in the bulk even at vanishing interaction.

The few-body peculiarity of the functional dependence of $\Delta$ on $g$ is confirmed by the Bethe-ansatz result for two paired fermions in a hard-wall trap of width $L[69]$. In fact, the spin-excitation gap $\Delta$ for the hard-wall confinement potential shown in Fig. 4(a) (dashed curve) almost matches that for the harmonic trap (solid curve) up to $g \sim-3$, provided that $L$ coincides with the harmonic oscillator length $\ell=(\hbar / m \omega)^{1 / 2}$. At small $g$ the gap scales like $\Delta \sim g^{2}$, which is clearly unrelated to the BCS-like functional dependence of Eq. (6).

\section{COOPER PAIRS}

To investigate pair formation we evaluate the conditional probability $P\left(x_{1}, x_{2}\right)$ of finding one atom at position $x_{2}$ with spin $\sigma_{2}=\uparrow$ if another atom is fixed at $x_{1}$ with opposite spin $\sigma_{1}=\downarrow$,

$$
P\left(x_{1}, x_{2}\right)=A \sum_{i, j=1}^{N}\left\langle\delta\left(x_{i}-x_{1}\right) \delta_{\sigma_{i}, \downarrow} \delta\left(x_{j}-x_{2}\right) \delta_{\sigma_{j}, \uparrow}\right\rangle,
$$

where the quantum average $\langle\ldots\rangle$ is taken over the ED ground state and $A$ is a normalization constant specified below. In Fig. 6 we choose $x_{1}$ as the average radius $x_{0}=\left\langle\left|x_{1}\right|\right\rangle$ (located by the red [gray] dot) and plot $P\left(x_{1}=x_{0}, x_{2}\right)$ versus $x_{2}$ (red [gray] curves). At small interaction strength $g=-0.45$ (dashed red [gray] curves) the conditional probability is essentially independent from the fixed atom position $x_{0}$, thus replicating the spin- $\uparrow$ noninteracting one-body density $\sum_{j}\left\langle\delta\left(x_{j}-x_{2}\right)\right\rangle$, whose peaks are Friedel oscillations induced by the harmonic confinement [58, 70]. For strong attraction, $g=$ -3 (solid red [gray] curves), $P\left(x_{0}, x_{2}\right)$ rearranges its weight, exhibiting a clear shrinking of the lateral extension together with a marked weight increase exactly at the position of the fixed atom. This suggests that the spin- $\uparrow$ atom at $x_{2}$ forms a bound Cooper pair with the spin- $\downarrow$ atom located at $x_{0}$.

To single out the internal structure of the Cooper pair we average $P\left(x_{1}, x_{2}\right)$ over the center-of-mass coordinate $X=\left(x_{1}+x_{2}\right) / 2$. The outcome is the pair correlation

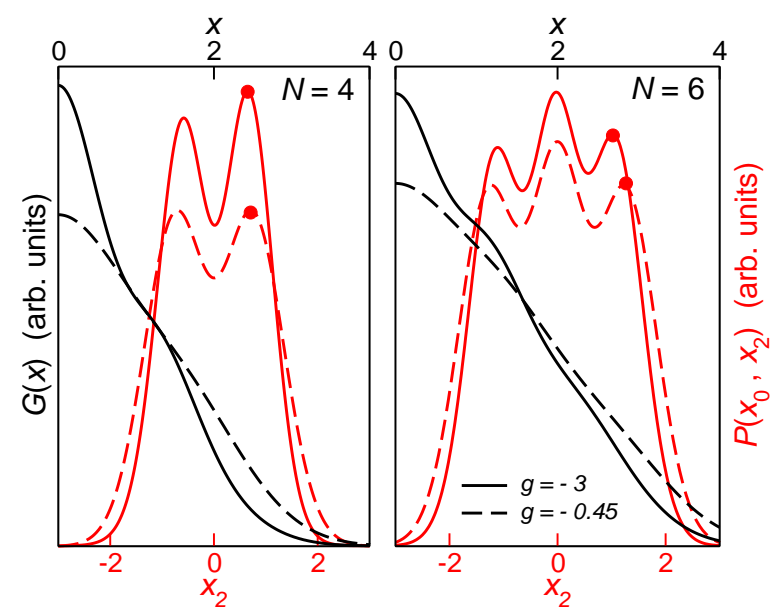

Figure 6. (color online) Conditional probability $P\left(x_{0}, x_{2}\right)$ vs $x_{2}$ (red [gray] curves, right and bottom axes) and pair correlation function $G(x)$ vs $x$ (black curves, left and top axes). Left and right panels concern $N=4$ and $N=6$, respectively. Dashed (solid) lines correspond to $g=-0.45(g=-3)$. Red [gray] dots locate the positions $x_{0}$ of spin- $\downarrow$ atoms. The length unit is $\ell=(\hbar / m \omega)^{1 / 2}$.

function

$$
G(x)=\int_{-\infty}^{\infty} d X P(X+x / 2, X-x / 2)
$$

which is the probability of finding two atoms of opposite spins at the relative distance $x=x_{1}-x_{2}$. We choose the normalization constant $A$ of (7) to obtain $\int d x G(x)=1$. Figure 6 shows that $G(x)$ develops a dominant peak at the origin, whose height increases with the interactionswitching from $g=-0.45$ (dashed black curves) to $g=$ -3 (solid black curves). This tendency maximizes the spatial overlap of two atoms with opposite spins while suppressing the probability of finding them to separately wander in the trap. Therefore $G(x)$ must be understood as the wave function square modulus of the Cooper pair in the frame of the relative distance $x$ between the two paired atoms, unrelated to the $X$-dependent distribution of pairs in the trap. Note that the counterpart of $G(x)$ in the bulk is the spatially-varying contribution to $P\left(x_{1}, x_{2}\right)$ explicited in (A4), both quantities rapidly vanishing as $\left|x_{1}-x_{2}\right| \rightarrow \infty$ [cf. Fig. 8 (f)].

The Cooper pair size $\xi$ may be immediately obtained as the quadratic displacement of $G(x)$,

$$
\xi_{G}^{2}=\int_{-\infty}^{\infty} d x x^{2} G(x) .
$$

We see in Fig. 7 that $\xi_{G}$ depends only weakly on the atom number (black symbols with solid lines) and decreases with increasing attraction, as the pair motion becomes more correlated. For comparison, we also evaluate the pair size $\xi$ through the BCS coherence length formula 15] (red [gray] symbols with dashed lines in Fig. 7), which in 


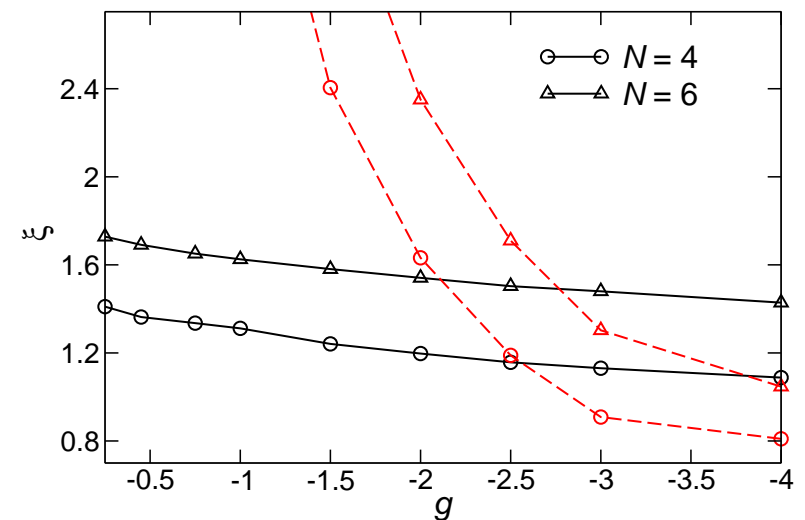

Figure 7. (color online) Cooper pair size $\xi$ vs interaction strength $g$ for $N=4$ (circles) and $N=6$ (triangles). Black (red [gray]) symbols with solid (dashed) lines point to the values of $\xi_{G}\left(\xi_{c}\right)$. We use $\hbar \omega$ as energy unit and $\ell=(\hbar / m \omega)^{1 / 2}$ as length unit, hence the dimensionless coupling constant is $g=g^{\prime} /(\hbar \omega \ell)$. The lines are guides to the eye.

standard units reads

$$
\xi_{c}=\frac{\hbar v_{F}}{2 \Delta}
$$

Here $v_{F}$ is the Fermi velocity obtained through the equivalence $m v_{F}^{2} / 2=\Delta_{1}^{*}(N)$ and $\Delta$ is taken from the ED excitation gap, $\Delta=E_{\text {exc }}(N) / 2$.

In the noninteracting limit obviously $\xi_{c} \rightarrow \infty$ (red [gray] symbols with dashed lines), whereas $\xi_{G}$ (black symbols with solid lines) tends to the natural limit fixed by the trap size. However, for $|g|>2$ the two estimates become comparable, pointing to a BEC-like regime where the pair size $\xi$ is smaller than both trap size and interparticle spacing, which could make correlated pair tunneling observable 22, 35]. This latter scenario contrasts with the nuclear case, where the size of nucleon pairs is larger than the system.

\section{REGIME OF STRONG ATTRACTION: PAULI BLOCKING IN REAL SPACE}

For strong inter-species attraction, the pair correlation function $G(x)$ develops one (two) shoulder(s) for $N=4$ $(N=6)$ [black solid curve for $g=-3$ in the left (right) panel of Fig. 6. This suggests that pairs arrange themselves in the trap to minimize the residual pair-pair repulsion due to exchange forces acting between atoms with parallel spins, as previously suggested in higher dimensions [56, 63, 71].

The shoulders in the pair wave function become more evident when dimers are strongly bound, as shown in Fig. 8 for $g=-4$. The pair size $\xi$ is now comparable to interparticle spacing, placing us on the BEC side of the BCS-BEC crossover (cf. Fig. 7). This is also seen

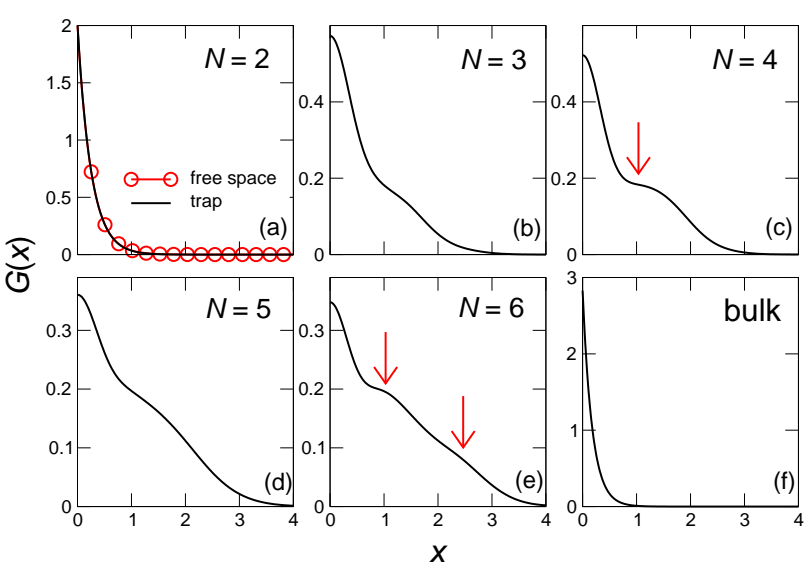

Figure 8. (color online) Pair correlation function $G(x)$ vs $x$ for (a) $N=2$ (b) $N=3$ (c) $N=4$ (d) $N=5$ (e) $N=6$ and (f) bulk at $g=-4$. Curves shown in panel a are analytical whereas those in panels b-e are computed using a basis set made of 50 (panel b) and 25 (panels c-e) harmonic-oscillator levels, respectively. The bulk curve of panel $\mathrm{f}$ is the spatiallyvarying part of $G(x)$ obtained from the BCS wave function. The length unit is $\ell=(\hbar / m \omega)^{1 / 2}$.

from the overlap between the wave function square modulus $G(x)$ in the trap [black line in Fig. 8 (a)] and in free space (circles) for a single pair, which is insensitive to the boundary as it is squeezed by interaction. Consistently, $\Delta=E_{\text {exc }}(N=2) / 2=2.23$ in the trap matches the expectation of Gaudin-Yang model for $\gamma \rightarrow-\infty$, which is half the binding energy of a single dimer, $\Delta=g^{2} / 8=2$.

Hovever, for more than one pair [Figs. 8 (b-e)] $G(x)$ qualitatively departs from the bulk prediction shown in Fig. $8(\mathrm{f})$ (derived in Appendix A). Whereas in the bulk $G(x)$ is a simple exponential, in the trap it displays $N / 2-1$ shoulders (with $N$ even), highlighted by arrows. While the first shoulder already appears for $N=3$ [Fig. 8(b)] this feature is significantly strengthened for $N=4$ [Fig. 8(c)], as the available number of Cooper pairs increases in a combinatorial fashion. Besides, as a second shoulder becomes evident for $N=6$ [Fig. 8(e)], the first shoulder moves closer to the origin. We attribute the overall behavior to Pauli blocking in real space, since two atoms of like spin cannot occupy the same trap orbital in the relative frame. This structure, peculiar to the trap, may be measured by time-of-flight spectroscopy 11].

\section{CONCLUSIONS}

In conclusion, we have studied a few 1D Fermi atoms in the presence of attractive contact forces through numerical exact diagonalization and found evidence of BCSlike paring. Whereas the present experiments may be understood by treating the interaction energy as a perturbation, we predict that non-trivial co-operative effects emerge at viable interaction strengths, when the Cooper 
pair size compares with the trap size.

\section{ACKNOWLEDGMENTS}

We thank Gerhard Zürn, Stephanie Reimann, Sven Åberg, Frank Deuretzbacher, and Nikolaj Zinner for stimulating discussions. This work is supported by EU-FP7 Marie Curie initial training network INDEX, MIUR-PRIN2012 MEMO, and CINECA-ISCRA grant IscrC_PAIR-1D.

\section{Appendix A: Bulk pair correlation function}

In this Appendix we derive the expression of the bulk pair correlation function $G(x)$ shown in Fig. $8(\mathrm{f})$. Throughout the Appendix we adopt standard units.

We introduce the BCS wave function $\left|\Psi_{\mathrm{BCS}}\right\rangle$ as the bulk ground state, being a standard variational ansatz in the whole range of the BCS-BEC crossover [6, 18, 72]. In second quantization, $\left|\Psi_{\mathrm{BCS}}\right\rangle$ takes the form

$$
\left|\Psi_{\mathrm{BCS}}\right\rangle=\prod_{k}\left(u_{k}+v_{k} \hat{c}_{k \uparrow}^{\dagger} \hat{c}_{-k \downarrow}\right)|0\rangle,
$$

where $\hat{c}_{k \uparrow}^{\dagger}$ is the fermionic operator that acts on the vacuum $|0\rangle$ creating an atom of spin $\uparrow$ and momentum $k$. As usual, the BCS coherence factors $u_{k}$ and $v_{k}$ occuring in (A1) are defined as

$$
u_{k}^{2}=\frac{1}{2}\left(1+\frac{\xi_{k}}{E_{k}}\right)
$$

with $u_{k}^{2}+v_{k}^{2}=1$. Here the quantity $\xi_{k}$ (not to be confused with the pair size $\xi$ ) is

$$
\xi_{k}=\varepsilon_{k}-\Delta_{1},
$$

with $\varepsilon_{k}=\hbar^{2} k^{2} / 2 m$ being the single-particle energy and $\Delta_{1}$ the bulk chemical potential, and the quasiparticle energy $E_{k}$ is

$$
E_{k}=\sqrt{\xi_{k}^{2}+\Delta^{2}}
$$

Depending on the value of the dimensionless coupling constant $\gamma=(g \pi / \hbar)\left(m / 8 \varepsilon_{F}\right)^{1 / 2}$, both chemical potentials $\Delta_{1}$ and pairing gap $\Delta$ should be determined simultaneously [72].

The bulk conditional probability $P\left(x_{1}, x_{2}\right)$ of finding one atom at position $x_{2}$ with spin $\uparrow$ if another atom is fixed at $x_{1}$ with opposite spin $\downarrow$, analogous to the definition (3) in the main text, is

$$
P\left(x_{1}, x_{2}\right)=\left\langle\Psi_{\mathrm{BCS}}\left|\hat{\Psi}_{\uparrow}^{\dagger}\left(x_{2}\right) \hat{\Psi}_{\downarrow}^{\dagger}\left(x_{1}\right) \hat{\Psi}_{\downarrow}\left(x_{1}\right) \hat{\Psi}_{\uparrow}\left(x_{2}\right)\right| \Psi_{\mathrm{BCS}}\right\rangle .
$$

Here $\hat{\Psi}_{\sigma}(x)$ is the annihilation field operator that destroys a fermion of spin $\sigma$ at position $x$ :

$$
\hat{\Psi}_{\sigma}(x)=\sum_{k} \frac{1}{\sqrt{L}} \mathrm{e}^{i k x} \hat{c}_{k \sigma},
$$

with $L$ being the system length. Inserting this expansion into (A2) and applying a standard manipulation, which parallels Appendix D of Ref. 16, we obtain:

$$
P\left(x_{1}, x_{2}\right)=\frac{N_{\uparrow} N_{\downarrow}}{L^{2}}+G\left(x_{2}-x_{1}\right),
$$

with $N_{\sigma}$ being the total number of atoms having spin $\sigma$. The conditional probability (A4) is the sum of a homogeneous background, $N_{\uparrow} N_{\downarrow} / L^{2}$, due to uncorrelated atoms having opposite spins, plus a spatially-dependent part, $G(x)$, which depends only on the relative distance $x=x_{2}-x_{1}$. Explicitly, one has:

$$
G(x)=\frac{\Delta^{2}}{16 \pi^{2}} \int d k \int d k^{\prime} \frac{\mathrm{e}^{i\left(k-k^{\prime}\right) x}}{E_{k} E_{k^{\prime}}} .
$$

This quantity may be regarded as the wave function square modulus of the Cooper pair.

We now focus on the strongly attractive regime of Fig. $8($ f). To proceed, we assume the pairing gap $\Delta$ to be the limit value for $\gamma \rightarrow-\infty$, i.e. half the binding energy of a single pair in free space, $\Delta=m g^{2} /\left(8 \hbar^{2}\right)$. In this limit we may neglect the contributions of $\varepsilon_{F}$ and interpair interactions to the chemical potential $\Delta_{1}$, hence $\Delta_{1}=-\Delta$ [72]. This allows us to expand the quasiparticle energy keeping only the linear term in $\varepsilon_{k}$, $E_{k} \approx \sqrt{\Delta_{1}^{2}+\Delta^{2}}\left[1-\Delta_{1} \varepsilon_{k} /\left(\Delta_{1}^{2}+\Delta^{2}\right)\right]$. Therefore, we may rewrite (A5) as $G(x) \propto I(x)^{2}$, where

$$
I(x)=\int d k \frac{\mathrm{e}^{i k x}}{k^{2}+k_{\mathrm{BCS}}^{2}}
$$

is the Fourier transform providing a decaying exponential, with

$$
k_{\mathrm{BCS}}=\frac{m|g|}{\sqrt{2} \hbar^{2}} .
$$

The final form of the normalized BCS pair wave function in the limit of strongly bound pairs, after dropping a prefactor, is:

$$
G(x)=k_{\mathrm{BCS}} \mathrm{e}^{-2 k_{\mathrm{BCS}}|x|},
$$

which is plotted in Fig. 8(f).

It is interesting to compare (A7) with the wave function square modulus $\left|\psi_{\text {dimer }}(x)\right|^{2}$ of a single pair in free space, which is shown in Fig. 8(a) (circles):

$$
\left|\psi_{\text {dimer }}(x)\right|^{2}=k_{\text {dimer }} \mathrm{e}^{-2 k_{\text {dimer }}|x|} \text {. }
$$

This has the same form as (A7) except for the decay length inverse,

$$
k_{\text {dimer }}=\frac{m|g|}{2 \hbar^{2}},
$$

which is a factor $\sqrt{2}$ smaller than $k_{\mathrm{BCS}}$. The shrinking of the pair size in the condensate is the effect of the exchange forces affecting the BCS many-body wave function [72]. 
[1] P. G. de Gennes, Superconductivity of metals and alloys (Westview Press, Boulder (Colorado), 1999)

[2] A. B. Migdal, Zh. Eksp. i Teor. Fiz. Pisma 37, 249 (1959), [Sov. Phys.-JETP 10, 176 (1960)]

[3] A. Bohr and B. R. Mottelson, Nuclear structure - Vol. $I$ and II (World Scientific, Singapore, 1998)

[4] V. L. Ginzburg and D. A. Kirzhnits, Zh. Eksp. i Teor. Fiz. Pisma 47, 2006 (1964), [Sov. Phys.-JETP 20, 1346 (1965)]

[5] D. Pines, G. Baym, and C. Pethick, Nature 224, 673 (1969)

[6] A. J. Leggett, Quantum liquids, 1st ed. (Oxford University Press, Oxford, 2006)

[7] M. Rontani and L. J. Sham, in Novel Superfluids Volume 2, International Series of Monographs on Physics, Vol. 157, edited by K. H. Bennemann and J. B. Ketterson (Oxford University Press, Oxford, UK, 2014) Chap. 19, pp. $423-474$

[8] C. A. Regal, C. Ticknor, J. L. Bohn, and D. S. Jin, Nature (London) 424, 47 (2003)

[9] M. Bartenstein, A. Altmeyer, S. Riedl, S. Jochim, C. Chin, J. H. Denschlag, and R. Grimm, Phys. Rev. Lett. 92, 120401 (2004)

[10] M. W. Zwierlein, J. R. Abo-Shaeer, A. Schirotzek, C. H. Schunck, and W. Ketterle, Nature (London) 435, 1047 (2005)

[11] I. Bloch, J. Dalibard, and W. Zwerger, Rev. Mod. Phys. 80, 885 (2008)

[12] S. Giorgini, L. P. Pitaevskii, and S. Stringari, Rev. Mod. Phys. 80, 1215 (2008)

[13] A. Bohr, B. R. Mottelson, and D. Pines, Phys. Rev. 110, 926 (1958)

[14] V. Zelevinsky and A. Volya, Phys. At. Nucl. 66, 1781 (2003)

[15] D. Brink and R. Broglia, Nuclear Superfluidity: Pairing in Finite Systems (Cambridge University Press, Cambridge (UK), 2005)

[16] J. Bardeen, L. N. Cooper, and J. R. Schrieffer, Phys. Rev. 108, 1175 (1957)

[17] C. Chin, R. Grimm, P. Julienne, and E. Tiesinga, Rev. Mod. Phys. 82, 1225 (2010)

[18] A. J. Legget, J. Phys. (Paris), Colloq. 41, C7 (1980)

[19] P. Nozieres and S. S. Rink, J. Low. Temp. Phys. 59, 195 (1985)

[20] F. Serwane, G. Zürn, T. Lompe, T. B. Ottenstein, A. N. Wenz, and S. Jochim, Science 332, 336 (2011)

[21] G. Zürn, F. Serwane, T. Lompe, A. N. Wenz, M. G. Ries, J. E. Bohn, and S. Jochim, Phys. Rev. Lett. 108, 075303 (2012)

[22] G. Zürn, A. N. Wenz, S. Murmann, A. Bergschneider, T. Lompe, and S. Jochim, Phys. Rev. Lett. 111, 175302 (2013)

[23] A. N. Wenz, G. Zürn, S. Murmann, I. Brouzos, T. Lompe, and S. Jochim, Science 342, 457 (2013)

[24] H. Moritz, T. Stöferle, K. Günter, M. Köhl, and T. Esslinger, Phys. Rev. Lett. 94, 210401 (2005)

[25] Y. Liao, A. S. C. Rittner, T. Paprotta, W. Li, G. B. Partridge, R. G. Hulet, S. K. Baur, and E. J. Mueller, Nature (London) 467, 567 (2010)

[26] K. Martiyanov, V. Makhalov, and A. Turlapov, Phys. Rev. Lett. 105, 030404 (2010)
[27] M. Feld, B. Fröhlich, E. Vogt, M. Koschorreck, and M. Köhl, Nature (London) 480, 75 (2011)

[28] P. Dyke, E. D. Kuhnle, S. Whitlock, H. Hu, M. Mark, S. Hoinka, M. Lingham, P. Hannaford, and C. J. Vale, Phys. Rev. Lett. 106, 105304 (2011)

[29] A. T. Sommer, L. W. Cheuk, M. J. H. Ku, W. S. Bakr, and M. W. Zwierlein, Phys. Rev. Lett. 108, 045302 (2012)

[30] H. Heiselberg and B. Mottelson, Phys. Rev. Lett. 88, 190401 (2002)

[31] G. M. Bruun and H. Heiselberg, Phys. Rev. A 65, 053407 (2002)

[32] H. Heiselberg, Phys. Rev. A 68, 053616 (2003)

[33] C. J. Pethick and H. Smith, Bose-Einstein Condensation in Dilute Gases (Cambridge University Press, Cambridge (UK), 2002)

[34] M. Rontani, Phys. Rev. Lett. 108, 115302 (2012)

[35] M. Rontani, Phys. Rev. A 88, 043633 (2013)

[36] J. von Delft and D. C. Ralph, Phys. Rep. 345, 61 (2001)

[37] L. Viverit, G. M. Bruun, A. Minguzzi, and R. Fazio, Phys. Rev. Lett. 93, 110406 (2004)

[38] H. Olofsson, S. Åberg, and P. Leboeuf, Phys. Rev. Lett. 100, 037005 (2008)

[39] M. M. Forbes, S. Gandolfi, and A. Gezerlis, Phys. Rev. Lett. 106, 235303 (2011)

[40] P. Ribeiro and A. M. García-García, Phys. Rev. Lett. 108, 097004 (2012)

[41] A. Angelone, M. Campostrini, and E. Vicari, Phys. Rev. A 89, 023635 (2014)

[42] M. Gaudin, Phys. Lett. A 24, 55 (1967)

[43] C. N. Yang, Phys. Rev. Lett. 19, 1312 (1967)

[44] M. Takahashi, Prog. Theor. Phys. 46, 1388 (1971)

[45] X.-W. Guan, M. T. Batchelor, and C. Lee, Rev. Mod. Phys. 85, 1633 (2013)

[46] K. Yang, Phys. Rev. B 63, 140511 (2001)

[47] T. Giamarchi, Quantum physics in one dimension (Clarendon, Oxford, 2003)

[48] M. A. Cazalilla, R. Citro, T. Giamarchi, E. Orignac, and N. Rigol, Rev. Mod. Phys. 83, 1405 (2011)

[49] G. E. Astrakharchik, D. Blume, S. Giorgini, and L. P. Pitaevskii, Phys. Rev. Lett. 93, 050402 (2004)

[50] I. V. Tokatly, Phys. Rev. Lett. 93, 090405 (2004)

[51] J. N. Fuchs, A. Recati, and W. Zwerger, Phys. Rev. Lett. 93, 090408 (2004)

[52] X. W. Guan, M. T. Batchelor, C. Lee, and M. Bortz, Phys. Rev. B 76, 085120 (2007)

[53] L. Guan, S. Chen, Y. Wang, and Z.-Q. Ma, Phys. Rev. Lett. 102, 160402 (2009)

[54] M. Rontani, C. Cavazzoni, D. Bellucci, and G. Goldoni, J. Chem. Phys. 124, 124102 (2006)

[55] S. Kalliakos, M. Rontani, V. Pellegrini, C. P. Garcia, A. Pinczuk, G. Goldoni, E. Molinari, L. N. Pfeiffer, and K. W. West, Nature Phys. 4, 467 (2008)

[56] M. Rontani, J. R. Armstrong, Y. Yu, S. Åberg, and S. M. Reimann, Phys. Rev. Lett. 102, 060401 (2009)

[57] A. Singha, V. Pellegrini, A. Pinczuk, L. N. Pfeiffer, K. W. West, and M. Rontani, Phys. Rev. Lett. 104, 246802 (2010)

[58] J.-J. Wang, W. Li, S. Chen, G. Xianlong, M. Rontani, and M. Polini, Phys. Rev. B 86, 075110 (2012)

[59] S. Pecker, F. Kuemmeth, A. Secchi, M. Rontani, D. C. 
Ralph, P. L. McEuen, and S. Ilani, Nature Phys. 9, 576 (2013)

[60] T. Sowiński, T. Grass, O. Dutta, and M. Lewenstein, Phys. Rev. A 88, 033607 (2013)

[61] P. D'Amico and M. Rontani, J. Phys. B: At. Mol. Opt. Phys. 47, 065303 (2014)

[62] O. Juillet, F. Gulminelli, and P. Chomaz, Phys. Rev. Lett. 92, 160401 (2004)

[63] J. von Stecher, C. H. Greene, and D. Blume, Phys. Rev. A 77, 043619 (2008)

[64] M. Casula, D. M. Ceperley, and E. J. Mueller, Phys. Rev. A 78, 033607 (2008)

[65] N. T. Zinner, K. Mølmer, C. Özen, D. J. Dean, and K. Langanke, Phys. Rev. A 80, 013613 (2009)
[66] C. N. Gilbreth and Y. Alhassid, Phys. Rev. A 88, 063643 (2013)

[67] The ED separation energy for $g=-0.9$ coincides with the one shown for $g=-1$ (black squares and solid lines) within $12 \%$.

[68] T. Busch, B. Englert, K. Rzążewski, and M. Wilkens, Found. Phys. 28, 549 (1998)

[69] Y. Hao, Y. Zhang, J. Q. Liang, and S. Chen, Phys. Rev. A 73, 063617 (2006)

[70] S. A. Söffing, M. Bortz, and S. Eggert, Phys. Rev. A 84, 021602(R) (2011)

[71] P. O. Bugnion, J. A. Lofthouse, and G. J. Conduit, Phys. Rev. Lett. 111, 045301 (2013)

[72] W. Ketterle and M. W. Zwierlein, La Rivista del Nuovo Cimento 031, 247 (2008) 